\title{
Characterising wheeze phenotypes to identify endotypes of childhood asthma, and the implications for future management
}

Danielle C. M. Belgrave MSc1,2, Adnan Custovic MD PhD1 and Angela Simpson MD PhD1

${ }^{1}$ Centre for Respiratory Medicine and Allergy, Institute of Inflammation and Repair, University of Manchester and University Hospital of South Manchester, Manchester, UK

${ }^{2}$ Centre for Health Informatics, Institute of Population Health, University of Manchester, UK 


\begin{abstract}
It is now a commonly held view that asthma is not a single disease, but rather a set of heterogeneous diseases sharing common symptoms. One of the major challenges to treating asthma is understanding these different asthma phenotypes and their underlying biological mechanisms. This review gives an epidemiological perspective of our current understanding of the different phenotypes that develop from birth to childhood that come under the umbrella term "asthma". The review focuses mainly on publications from longitudinal birth cohort studies where the natural history of asthma symptoms is observed over time in the whole population. Identifying distinct pathophysiological mechanisms for these different phenotypes will potentially elucidate different asthma endotypes, ultimately leading to more effective treatment and management strategies.
\end{abstract}

Keywords: asthma phenotypes, endotypes, epidemiology, machine learning, longitudinal cohorts

\title{
Introduction
}

The increasing global prevalence of childhood asthma is of major concern[1]. One of the challenges to understanding the pathophysiology and aetiology of this disease is the lack of consensus in defining asthma in the medical literature. A recent systematic review showed that in 122 publications investigating risk factors associated with childhood asthma, 60 different definitions were used[2]. Although many of these definitions were similar (with apparently subtle differences between them), the impact of this heterogeneity on the reported results and our understanding of asthma is unclear. When four of the most commonly used definitions were applied to a high risk population of 186 children, the overall agreement was $61 \%$, indicating that $39 \%$ of the children could move from 'asthma' to 'not asthma' depending on which definition was used[2]. Such variability will obviously affect the performance of prediction models. It is clear that better tools for the diagnosis of asthma are required, both for clinical practice and for epidemiological studies. Throughout the remainder of this review, we will present evidence to support our view that "asthma" is not a single 
disease but rather represents an umbrella term used to describe a collection of heterogeneous diseases presenting with similar symptoms[3] such as recurrent episodes of wheeze, cough, breathlessness and chest tightness[4]. We will also explain the importance of identifying and describing these separate entities. For many of our patients, asthma starts early in life, therefore we have focused this review on paediatric studies, in particular birth cohorts. For many others asthma starts in adulthood, and although this can be severe and is certainly heterogeneous, a summary of that literature is beyond the scope of the current review.

Asthma runs in families and is clearly heritable, but patterns of inheritance are complex and genetic studies have shown poor replication, and loci which have been replicated generally explain a very small proportion of the heritability, with small effect sizes $[5,6]$. The rapid increase in the prevalence of asthma seen in the later part of the last century strongly indicates that environmental exposures are highly relevant in the aetiology of wheezing illnesses. Despite several decades of extensive epidemiological study, we are still unable to explain the increase in prevalence, and findings are inconsistent for most environmental factors. An example of an inconsistent association with asthma is day-care attendance. Different studies have indicated that day-care attendance increases the risk[7], is protective[8] and has no effect[9] on the development of asthma. It is likely that imprecise phenotype definition together with the failure to take account of interactions between the environment and genetic predispositions (day care - TLR2, endotoxin - CD14 being but two examples) [10-12] have hampered our attempts to 'explain' asthma. In order to progress our field of knowledge, and to design targeted and effective treatments for our wheezing patients, we need to precisely define the diseases under the "asthma umbrella", and then determine their genetic and environmental predictors. Whereas phenotype is indicative of the observable characteristics or symptoms of a disease, an endotype refers to "a subtype of a condition, which is defined by a distinct functional or pathophysiological mechanism"[13]. Disentangling the different phenotypes that are generically described as "asthma" is an important step towards describing true endotypes of asthma with distinct underlying pathophysiological mechanisms[13]. Defining distinct endotypes of 
asthma (which may have different causes) will facilitate more precise treatment and management strategies for these different diseases.

Although wheeze is the most commonly observed manifestation of asthma, it has long been recognised that all that wheezes is not asthma, particularly in pre-school age, and that distinctions are important in terms of therapeutics[14]. Furthermore, sequential cross-sectional studies of populations of children from school age will never allow a description of the within individual natural history of wheezing illness. The existence of longitudinal observational birth cohorts which record the natural history of asthma-related symptoms over time such as wheeze and cough have allowed us to describe patterns in the rich and complex datasets, to classify children based on the frequency and severity of symptom progression from birth through early childhood.[15-18] Understanding the heterogeneity or different manifest patterns of wheeze over time (wheeze phenotypes), may help us to differentiate different phenotypes which present under the umbrella term of "asthma" (asthma phenotypes) whilst acknowledging that not all phenotypes of asthma have wheeze as the dominant symptom (for example cough-variant asthma). Causative factors of recurrent wheeze may vary from child to child, and within a child over time due to a large number of interactions between genetic and environmental factors.[19,20] By identifying homogenous groups of children with similar disease features, we hope to better understand the development, comorbidities and associated genetic and environmental risk factors for these entities. Ultimately, this will have major implications for the effective treatment, prevention and management of this disease which is of increasing global concern[21].

\section{Towards a Definition of the Asthma Phenotype}

We can divide the approaches to classifying children according to distinct asthma phenotypes as 1) a subjective hypothesis-driven approach whereby the investigator looks at individual changes in the profile of asthma symptoms throughout childhood and then classifies children into different 
phenotypes based on common patterns of symptoms over time and 2) a data-driven or hypothesisgenerating approach, whereby the investigator uses latent variable modelling techniques. Hierarchical clustering is a latent variable modelling technique which allows us to infer that we can partition homogeneous groups of patients into meaningful clusters. It is assumed that different clusters have different probability distributions and patients with similar probability distributions form a cluster. Hierarchical clustering can be used to classify profiles of symptoms over time which are continuous (such as lung function) or a mixture of continuous and categorical data. Latent class analysis (LCA) is a type of hierarchical clustering which is used when we have categorical data or a binary diagnosis (i.e. symptom present or symptom absent). This is especially useful where we have multiple dichotomous symptoms, such as wheeze, which is tracked over time. LCA is a statistical method used when we infer that some latent generalisation or hypothetical construct, exhibits certain observable characteristics. This approach is widely applied in the clinical psychiatry literature [22-26] where investigators try to measure the severity of conditions which are not directly observed such as depression, intelligence or schizophrenia. These conditions are not directly measurable, but rather severity can be represented using a set of discrete latent constructs which can be derived based on a series of observable disease manifestations. In the same way, we can think of different childhood asthma phenotypes as latent states whose existence is made manifest by the longitudinal pattern of wheeze and/or other respiratory symptoms over time. We assume that each child belongs to one of $\mathrm{N}$ latent classes, where the number and size of these latent classes is unknown. Different assumptions of the number of classes, $\mathrm{N}$, are investigated in the model and the models are compared for goodness-of-fit to select the optimal number of latent profiles which best describe the observed heterogeneity in asthma symptoms between children over time. These probability-based modelling techniques gives us an objective method of assigning children to different latent asthma phenotypes with a robust evaluation of model reproducibility.

Within the literature, the most common approach used for identifying phenotypes of childhood asthma is to look at the natural history of wheeze over time, since this is the most easily measurable 
symptom of asthma. This mimics the real-life scenario whereby a history of recurrent wheeze is very often used to make a clinical asthma diagnosis. Although many other symptoms are related to asthma such as cough, shortness of breath and chest tightness there has been lack of reproducibility and consensus in identifying the best subgroup of symptoms to identify asthma phenotypes. This further justifies the use of recurrent wheeze which is by clinical consensus the most common manifestation of asthma. Therefore, this review focuses mainly on understanding wheeze phenotypes by looking for heterogeneous patterns of wheeze over time. Such an approach helps us to disambiguate the complex patterns of childhood wheeze.

\section{Subjective Approaches to Defining Wheeze Phenotypes}

Martinez et al in the Tucson Children Respiratory Study (TCRS) presented the first longitudinal classification of children into distinct wheeze phenotypes, based on the clinical assessment of the natural history of wheeze in the first six years of life. Based on these longitudinal patterns in 826 children, the authors assigned each child to one of four categories according to their history of wheezing: (1) children who never wheezed (51\%), (2) transient early wheezing: these children presented with recurring wheeze till age three years, but grew out of wheeze by age six years (20\%), (3) late-onset wheezing: these children did not wheeze in the first three years of life and then presented with wheeze at age six years (15\%) and (4) persistent wheezing: these children had an onset of wheeze in early life which persisted to age six years (14\%).[27] This classification identified that more than half of infant wheezing was transient, associated with diminished airway function in infancy, and was not associated with risk of asthma or allergies in later childhood. A number of birth cohorts have used similar approaches of investigator-imposed classifications to define and investigate clinical associations of different wheeze phenotypes[28]. This has led to the description of phenotypes of wheeze with similar names but differing definitions, making comparisons across cohorts challenging. $[4,29-33]$ 
The Avon Longitudinal Study of Parents and Children (ALSPAC), was the first study to consider the use of a probabilistic data-driven approach to classify children into different wheeze phenotypes. This study used latent class analysis to assign 6265 children to different phenotypes based on the longitudinal patterns of wheezing within the first seven years of life. They identified an optimal modelling solution of six phenotypes based on model goodness-of-fit statistics. By observing the prototypical pattern of wheeze over time for each class, the investigators labelled them as: (1) Never/infrequent wheeze (59\%) (2) intermediate onset wheeze (3\%): children who had no wheeze in the first year of life, but had an increasing probability of wheeze from ages two and a half years to age seven (3) transient early wheeze: children who wheezed in the first two and a half years of life, but grew out of wheeze between ages three and four years (16\%) (4) prolonged early wheeze: children who grew out of wheeze by age six years (9\%) (5) late onset wheeze: children who had a low probability of wheeze in the first three years of life, with increasing probability of wheeze between ages four and seven years (6\%) and (6) children with persistent wheeze who had a high probability of wheeze throughout childhood (7\%).[34] This data-driven approach with a larger study sample identified the three wheezing phenotypes described using the TCRS data, but detected two novel wheeze classes (intermediate and prolonged early).

An important validation of this data-driven approach to defining wheeze phenotypes has been the replication of these findings in the Dutch Prevention and Incidence of Asthma and Mite Allergy (PIAMA) cohort.[35] This study which has similar data-collection points to ALSPAC found evidence of the novel intermediate-onset wheeze phenotype. Phenotypes identified had similar clinical characteristics to ALSPAC and the probability of membership to the different wheeze classes was also similar. However, the optimal model was a five-latent-class solution which did not identify the prolonged early wheeze phenotype. In a later study, it was hypothesised that since prolonged early and transient early have very similar characteristics and risk factors, prolonged early wheeze may be 
a more severe form of transient early wheeze.[36] By using an objective data-driven approach across two geographically distinct cohorts, the investigators were able to derive broadly similar latent classes of wheeze with similar associations.

Chen et al[37] used similar latent modelling techniques to identify phenotypes of 689 children from the Columbia Center for Children's Environmental Health study (CCCEH) age 3 to 108 months using more complex assumptions of changes in the natural history of wheeze over time. They identified four classes of children, (never/infrequent, early transient, early persistent and late onset) similar to the TRCS study.

One of the problems with identifying wheeze phenotypes in longitudinal birth cohorts is that presence of wheeze is often based on parental reporting of wheeze. We have previously shown that a high proportion (approximately one third) of parentally reported wheeze was not confirmed on physician consultation[38]. The confirmation of wheeze by a doctor may give a more accurate judgment on the presence (and severity) of wheeze. Incorporating information on wheeze from both parental and clinical sources can elucidate both dimensions of uncertainty and severity of the asthma endotype. Both sources provide important information and are complimentary - it is recognised that parents may misdiagnose wheeze, so the physician report is an important confirmation in early life[38]; conversely treatment may suppress wheeze leading to underreporting of events by parents. A further complicating factor is that parents may not always consult a primary care physician for non-severe wheeze events. Using longitudinal latent class modeling[39] of questionnaire data completed by parents and the data transcribed from primary care medical records on wheeze within the first eight years of life, we have recently investigated longitudinal patterns of wheezing amongst children in the Manchester Asthma and Allergy Study (MAAS)[40]. This approach of joint modelling of 'higher resolution' data pertaining to wheeze events, allowed us to model uncertainty in parental or physician diagnosis of wheeze. We identified four classes of wheeze (in addition to a non-wheezing class)[40]. Based on our interpretation of their characteristics, we named two of the classes transient early-wheeze, and late onset wheeze, similar 
to TCRS. However, our model identified two classes of children with persistent wheeze, and based on their consulting behaviour and requirements for asthma treatment, we named them Persistent troublesome and Persistent controlled wheeze[40]. So, how similar are the findings between different methods and different cohorts? Using data collected for in the Southampton's Women's Survey (SWS) from 1973 children (born to $>12,000$ women recruited pre-conception) and followed prospectively (including infant lung function in 150), researchers have assigned children to the TCRS classes and also to the ALSPAC classes[41]. They concluded that a six class (ALSPAC) model better reflected their data. We have compared the proportion of children in each class when the TCRS phenotypes are applied (Tables $1 \mathrm{~A}$ and B) and also the proportions assigned to each class for data driven approaches (Table $2 \mathrm{~A}$ and B). All cohorts, whether using objective or subjective classification methods identified the four common classes: No Wheeze, Transient-early Onset, Late-onset wheeze and Persistent Wheeze. However the proportion of children assigned to each class varies by cohort. There is up to 10 -fold variation in the proportion of wheezing children who are assigned to late onset wheeze defined according to TCRS criteria (Table 1B), but a similar variability in the size of the late onset class is seen when data driven approaches are applied (Table 2B). Although this may in part reflect the time points at which data was collected and the differing characteristics of the cohorts, it does indicate that further work is required before the definitive phenotypes are identified.

Table 1: Summary of wheeze phenotypes identified in some birth cohorts with proportion of children assigned to each class using the TCRS classification for A) the whole population, and B) the proportions amongst wheezing children.

\section{Table 1A}

\section{Phenotypes Identified}

TCRS [16]
MAAS [28] (to age 5 years) (to age 7 years) 


\begin{tabular}{lcccc}
\hline No Wheeze & $51.5 \%$ & $40.3 \%$ & $55.6 \%$ & $64.8 \%$ \\
\hline Transient Early Wheeze & $19.9 \%$ & $45 \%$ & $23.5 \%$ & $25.0 \%$ \\
\hline Late-Onset Wheeze & $15.0 \%$ & $2.2 \%$ & $5.8 \%$ & $5.3 \%$ \\
\hline Persistent Wheeze & $13.7 \%$ & $12.5 \%$ & $15.1 \%$ & $4.9 \%$ \\
\hline
\end{tabular}

Table 1B

\begin{tabular}{lcccc}
\hline Phenotypes Identified & TCRS [16] & SWS [41] & $\begin{array}{c}\text { MAAS [28] } \\
\text { (to age } 5 \text { years) }\end{array}$ & $\begin{array}{c}\text { MAS } 90 \text { [30] } \\
\text { (to age } 7 \text { years) }\end{array}$
\end{tabular}

\begin{tabular}{lcccc}
\hline Transient Early Wheeze & $41.0 \%$ & $75.4 \%$ & $52.9 \%$ & $71.0 \%$ \\
\hline Late-Onset Wheeze & $30.9 \%$ & $3.7 \%$ & $13.1 \%$ & $15.1 \%$ \\
\hline Persistent Wheeze & $28.2 \%$ & $20.9 \%$ & $34.0 \%$ & $13.9 \%$ \\
\hline
\end{tabular}

Table 2: Proportion of children assigned to each class using data driven analysis for $A$ ) the whole population, and B) the proportions amongst wheezing children

Table 2A

\begin{tabular}{|c|c|c|c|c|c|}
\hline Phenotypes Identified & ALSPAC [34] & PIAMA [35] & MAAS [40] & CCCEH [37] & SWS [41] \\
\hline No Wheeze & $61.1 \%$ & $75.0 \%$ & $53.3 \%$ & $47.1 \%$ & $40.3 \%$ \\
\hline Transient Early Wheeze & $16.5 \%$ & $16.7 \%$ & $13.7 \%$ & $37.5 \%$ & $17.4 \%$ \\
\hline Prolonged Early Wheeze & $9.3 \%$ & & & & $27.6 \%$ \\
\hline Intermediate Onset Wheeze & $2.5 \%$ & $3.1 \%$ & & & $6.2 \%$ \\
\hline Late-Onset Wheeze & $4.9 \%$ & $1.7 \%$ & $16.7 \%$ & $7.8 \%$ & $2.2 \%$ \\
\hline
\end{tabular}




\begin{tabular}{lcccc}
\hline Persistent Wheeze & $5.8 \%$ & $3.5 \%$ & & $7.6 \%$ \\
Persistent Troublesome Wheeze & & $3.2 \%$ & \\
& & $13.1 \%$ & \\
Persistent Controlled Wheeze & & & \\
\hline
\end{tabular}

Table 2B

\begin{tabular}{|c|c|c|c|c|c|}
\hline Phenotypes Identified & ALSPAC [34] & PIAMA [35] & MAAS [40] & CCCEH [37] & SWS [41] \\
\hline Transient Early Wheeze & $42.4 \%$ & $66.8 \%$ & $29.3 \%$ & $70.9 \%$ & $29.1 \%$ \\
\hline Prolonged Early Wheeze & $23.9 \%$ & & & & $46.2 \%$ \\
\hline Intermediate Onset Wheeze & $6.4 \%$ & $12.4 \%$ & & & $10.4 \%$ \\
\hline Late-Onset Wheeze & $12.7 \%$ & $6.8 \%$ & $35.8 \%$ & $14.9 \%$ & $3.68 \%$ \\
\hline Persistent Wheeze & $14.9 \%$ & $14.0 \%$ & & $14.5 \%$ & $10.7 \%$ \\
\hline Persistent Troublesome Wheeze & & & $6.9 \%$ & & \\
\hline Persistent Controlled Wheeze & & & $28.1 \%$ & & \\
\hline
\end{tabular}

\section{Characteristics and Risk Factors Associated with Wheeze Phenotypes}

Understanding the characteristics and risk factors of different wheeze phenotypes can potentially elucidate asthma endotypes with distinct causal pathophysiological mechanisms[42]. This in turn can facilitate research towards identifying more effective personalised treatment and management strategies in accordance with the nature of the endotype. It is acknowledged that this is a highly complex area. Wheezing in infants and later development of asthma may be the result of alterations of the immune system maturation and acquired modifications of the airway in early life[43,44]. This is likely controlled by factors including genetics, epigenetics, environmental exposures and geneenvironment interactions[12]. In this part of the review, we summarise our current understanding of 
the characteristics of and some risk factors associated with the different wheeze phenotypes identified. Table 3 gives an overview of these characteristics and risk factors.

\section{Early life wheeze (transient and prolonged)}

Approximately half of all children who wheeze in early life have transient symptoms which do not persist in later childhood. Although the original Tucson phenotypes suggested that transient early wheeze resolved by age 3 years, this may in part reflect the ages of the children when the data was collected (0-3 years and 5-6 years). The data driven approach from ALSPAC suggested that in addition to a transient early wheeze (TEW) group (in whom symptoms had largely resolved by 3 years), there was a smaller, second group "prolonged early wheeze" (PEW) in whom wheeze persisted to $\sim 5$ years of age. This group would not have been detected as a separate entity in TCRS because of time points of data collection, and may reflect the very large ALSPAC dataset (with data collected at 7 time points in seven years). In the PIAMA dataset, the prolonged early wheeze class was not identified as a separate entity, but the PIAMA TEW group seemed to reflect a combination of the ALSPAC TEW and PEW both in terms of size (accounting for $~ 70 \%$ of children who had wheezed, Table 2B) and of time course of prevalence of wheeze. It is of note that the PIAMA cohort is significantly smaller in size than ALSPAC. In the SWS compared to TCRS, many more of the SWS children had TEW, and fewer had LOW (Table 1B). Interestingly, when the ALSPAC latent classes were applied to the SWS, again the combination of TEW and PEW accounted for $\sim 70 \%$ of the children (although in SWS, PEW was more common than TEW, Table 2B).

It was noted within TCRS that those children with TEW had significantly lower early life lung function (measured before any wheeze event, at age 2 months) than all other groups[27], and that this defect persisted to age six years. Within SWS, using the Tucson classification TEW children, early life lung function was diminished compared to the never wheezers, but this defect was no longer detectable by age 6 years[41]. In contrast, in MAAS TEW was significantly associated with diminished lung function at age 5 years, but not at age 3 years[28]. Within ALSPAC there is no data available on lung 
function in infancy, but when SWS assigned children to the ALSPAC wheeze phenotypes, lung function in infancy was diminished in the PEW, and this abnormality in lung function persisted to age six years. These data do suggest that early life wheezing is associated with diminished lung function prior to the onset of wheeze; however this phenomenon was not seen in a cohort of Australian children who had stopped wheezing by age 3 years[33]. It should be noted however that because of the technical difficulties in performing lung function in infancy these studies tend to be small.

Within ALSPAC and PIAMA (where data on infant lung function was not presented), TEW and PEW groups had diminished lung function at age 6-8 years (i.e. after wheezing had resolved) compared to never wheezers. Airway hyperresponsiveness was either not associated $[35,41]$ or weakly associated with membership of this class[34,40]. Allergic sensitisation was not a feature of TEW or PEW groups, and neither was eczema or rhinitis associated with these groups.

Exposure to tobacco smoking has been associated with TEW in all studies where this has been investigated $[27,36,41]$. Exposure to tobacco smoke is also a risk factor for other wheeze phenotypes that start early in life (in some but not all studies), but is less consistently associated with late onset wheeze. Attending day care has been identified as a risk factor for TEW in three studies. Position in sibship and male gender were not consistent across the cohorts [45-47]. Early wheeze is not associated with breast feeding, birth weight or BMI, but (like all wheeze phenotypes) is more common in those whose mothers have asthma.

It is likely that much early wheeze is virally induced [47]. Many children develop symptoms of wheeze during early life infection with respiratory syncytial virus (RSV) and observations from population based birth cohorts suggest that these children may be at an increased risk of asthma in later childhood $[48,49]$. However, by collecting nasopharyngeal aspirates during and between acute respiratory illnesses in a high risk cohort, Jackson et al were able to demonstrate that children who wheezed only with RSV during infancy were no more likely to develop asthma by age 6 years than those who did not wheeze with RSV (or rhino virus-RV). In contrast, children who wheezed with RV 
during infancy were at an increased risk of asthma at age 6 years compared to children who did not wheeze with RV (or with RSV)[50]. Until molecular mechanisms which underlie these responses are elucidated, and genetic predispositions are deciphered, the conflicting results of epidemiological studies remain difficult to understand. In the case of RSV, where treatments are available, the true effects of RSV on wheezing illness in childhood will not be clarified until the results of randomised, placebo-controlled clinical trials which test whether the prevention of RSV lower respiratory illness reduces the incidence of subsequent asthma[51].

Although the use of ICS for early wheezing illness does not change the natural history of asthma or wheeze in later childhood[52], and the effect of ICS on symptoms in infants with wheezing is minimal at best[52,53], many children within transient wheeze classes received inhaled steroids (ICS) in early life[35,40]. The transient wheezing group accounts for most of the preschool children receiving ICS[40], suggesting over-treatment in this group of children. This is potentially a non-trivial misdiagnosis as it has been previously shown that intermittent high dose ICS may affect normal growth and development[54].

\section{Persistent Wheeze (troublesome and controlled)}

In all cohorts where this has been investigated, persistent wheeze is characterised by diminished lung function by school age $[27,28,30,34,41]$. Very little infant lung function data is available, and it is not clear whether lung function soon after birth is diminished in this group, as results are inconsistent between studies[27,41]. In MAAS, children with persistent wheeze fell into two separate classes (persistent troublesome wheeze and persistent controlled wheeze[40]); the persistent troublesome wheeze group had materially poorer lung function and more reactive airways than other groups, including the persistent controlled wheeze group[40]. By using sRaw (Specific Airway Resistance) as a measure of lung function it was also possible to see that 
longitudinal lung function is significantly poorer in the persistent troublesome wheeze compared to all other phenotypes, and this effect was seen from age 3 years[40]. Persistent troublesome wheezers were also most likely to show bronchial hyperresponsiveness, but again this feature is common to other wheeze phenotypes[40].

More than $50 \%$ of children in the persistent wheeze class are atopic[47]. However, although this is not a feature of transient wheeze, late onset and intermediate onset wheeze phenotypes also frequently show this characteristic and so in isolation, this is not a good discriminator of wheeze class $[27,29,35,36,45]$. In a study looking at defining different dimensions of atopy, we found that the latent class of multiple early sensitisation was significantly associated with persistent wheeze compared to all other wheeze phenotypes[55]. This indicates that not only is asthma and wheezing illness a heterogeneous disease with distinct longitudinal profiles in childhood, but also there is also a similar underlying latent heterogeneity in atopy[55,56]. Similar to asthma, defining atopy as a dichotomous trait may be an oversimplification of this phenotype.

As with other wheeze phenotypes, maternal history of asthma and gender are significantly associated with the risk of persistent wheeze. However, for other associates the data is less consistent. Lower socio-economic status was associated with both persistent and early wheeze phenotypes in both ALSPAC and SWS, with no significant association with later-onset wheeze phenotypes. While SWS found that breastfeeding for less than three months was a significant risk for persistent wheeze[41] compared to transient-early wheeze, this association has not been replicated in any other study. In the TCRS, this association was found to be confounded by maternal asthma.[9,57]

There was lack of agreement with regards to the association of day-care with persistent wheeze, with ALSPAC finding that day-care was associated with an increase in persistent wheeze[36], TCRS finding that day-care was protective [27]. Similarly, ALSPAC identified a significant positive association between persistent wheeze and number of older siblings[32,36], whereas TCRS found 
that this was the only wheeze phenotype where the presence of older siblings reduced the risk of persistent wheeze[27].

\section{Late-onset Wheeze}

Late-onset wheeze is generally characterised as wheeze-onset observed after age three years and then persists to later childhood. There is lack of agreement across studies with respect to the association of late-onset wheeze with impaired lung function and bronchial hyperresponsiveness. ALSPAC[34], PIAMA[35] and MAAS[40] all found that children with late-onset wheeze were significantly more likely to have bronchial hyperresponsiveness. However, the Southampton Women's Study (using both the TCRS and ALSPAC definitions) found no significant association of late-onset wheeze with bronchial hyperresponsiveness[41]. MAAS and ALSPAC found significant association of late-onset wheeze with lung function impairment at age $6[28,34]$, whereas PIAMA, SWS and TCRS found no significant association for either early or late lung function impairment. The main characteristic which is consistently associated with late-onset wheeze is sensitisation, both before and concurrent with the onset of wheezing events.

Observational studies have generally found no environmental factors associated with later-onset wheeze phenotypes[27,34-36,41]. Two studies identified pre-natal smoking as a risk factor for lateonset wheeze[27,32]. This result was not replicated in other cohorts[34-36,41].

To our knowledge, there is currently no literature available on the history of asthma-related symptoms followed up from birth into adulthood. Extensions of current longitudinal birth cohort studies into adolescence and adulthood may elucidate whether this late-onset wheeze phenotype persists into adulthood or if transient wheezing becomes dormant over a limited period of longitudinal observation in childhood and then is retriggered in later life.

Overall, there are many similarities between the findings of the different studies of wheeze phenotypes indicating some reproducibility, whilst acknowledging that there are some clear 
differences. Further studies of this type will allow future reviewers to draw more firm conclusions as to the consistent and replicable phenotypes, which are more likely to indicate discrete endotypes. In addition, it is important to consider other features of asthma and to develop more complex models.

\section{More Complex Models: Dimensions of Respiratory Disease in Childhood}

The studies reviewed so far have investigated profiles of recurrent wheeze over time as the main marker to establish asthma heterogeneity in childhood. The data-driven approaches described can be extended to identify the most relevant symptoms and factors to distinguish between children with different phenotypes and disease severity. Syndromes of coexisting symptoms may better reflect the underlying pathophysiological processes and persistence of asthma symptoms in childhood [58]. Identifying groups of symptoms which coexist for different asthma phenotypes will improve diagnosis and identification of patients who respond to treatment. Previous studies have looked at understanding the different dimensions of respiratory diseases[58-61]. Although these studies are not limited to asthma, they are useful for helping us to distinguish comorbidities of different asthma phenotypes.

\section{Defining wheeze phenotypes within a clinical setting}

Two recent studies - the Severe Asthma Research Program (SARP) and the Trousseau Asthma Program in Paris (TRAPP) - have defined heterogeneous asthma phenotypes using cross-sectional data from a clinical population of asthmatics. Although these studies are cross-sectional in nature and therefore do not look at the natural history of asthma development for early identification and onset of phenotypes, they are useful for shedding light on the implications of data-driven techniques within a clinical setting, and were able to take into account other symptoms of asthma severity 
besides wheeze. In a study of asthmatic children between ages 6 and 17 years, SARP[62] identified 4 clusters labelled as 1) late-onset symptomatic asthma (29.8\%): these children had normal lung function and less atopy than all other asthmatics; 2) early-onset atopic asthma with normal lung function (32.2\%); 3) early-onset atopic asthma with mild airflow limitation and comorbidities (19.9\%) who had the longest asthma duration; and 4) early-onset asthma with advanced airflow limitation (18.1\%) which differed from 3 ) in terms of ethnicity (this class had children of mainly African descent whereas class 3 was mainly Hispanic), had the highest eNO values, but fewer comorbidities compared to class 3). Using similar statistical techniques, TRAPP[63], a study of children between the ages of 6 and 12 years, identified two novel severe asthma phenotypes by including inflammatory markers in their cluster analysis, distinguishing between 1) asthma with severe exacerbations and multiple allergies (32.7\%); 2) severe asthma with bronchial obstruction (22.9\%) and 3) mild asthma (44.4\%). Both studies found poor correlation between the GINA guidelines for classifying asthma severity and the asthma phenotypes identified with the respective study. Furthermore, the asthma phenotypes identified were better predictors of disease severity compared to GINA. This provides evidence of the potential clinical relevance of these data-driven approaches to classifying children.

\section{Strategies for Predicting Asthma Development}

The first three years of life are important in childhood development and the later onset of respiratory disease. Of particular interest is the early identification of children who are early wheezers and distinguishing between those who will go on to persist in wheezing symptoms from those who will grow out of symptoms. This is important for distinguishing between a wheeze phenotype which is of a transitory nature in early childhood and wheeze of a more persistent nature which may be more in line with current definitions of asthma. Due to the inherent difficulty in diagnosing children as "asthmatic" in early life, this may be pivotal in identifying possible earlyintervention strategies for reducing the risk of the later-onset of asthma. The Pollution and Asthma 
Risk: An Infant Study (PARIS) birth cohort recently identified three distinct phenotypes within the first 18 months of life[64]: (1) a mild phenotype (17\%), an atopic-severe phenotype (3.2\%) and a non-atopic severe phenotype (11\%), with the remaining $69 \%$ of children classified as having no recurrent wheeze events. Only with long-term follow up will it become clear whether the wheeze classes identified early in life are of prognostic value.

The Asthma Predictive Index (API) has been used as a tool to predict which children with early-onset wheeze will go on to develop persistent symptoms, and to distinguish them from children whose wheeze is of a transient nature. A positive API is given to a child in the first three years of life if they have recurrent episodes of wheezing during the previous years in addition to one out of two major criteria (physician-diagnosed eczema or parental asthma) or two out of three minor criteria (physician-diagnosed allergic rhinitis, wheezing without colds, or peripheral eosinophilia).[65] A positive API by age 3 is associated with a 76\% chance of active asthma from age 6-13 years, compared with $<5 \%$ chance of active asthma in the school years in those with a negative API at age 3. Other strategies have been suggested that, although they do not have as good a predictive ability as the API, can be used as complementary approaches for identifying persistent wheeze endotypes.[66] The PIAMA study suggests 8 clinical parameters for the risk score: male sex, postterm delivery, parental education and inhaled medication, wheezing frequency, wheeze/dyspnoea apart from colds, respiratory infections, and eczema are independent factors to predict subsequent asthma.[67] However, to date, the API has been proposed as the best, most practical strategy for predicting children who will develop persistent wheeze.[66]

Our data suggest that at age 3 years, predictors of subsequent troublesome symptoms amongst children with wheezing were large total skin test wheal diameter $(\geq 10 \mathrm{~mm})$, history of previous exacerbations, diminished lung function and current eczema.[40] 


\section{Pathophysiological Characteristics and Biomarkers: From Wheeze}

\section{Phenotypes to Asthma Endotypes}

One area of particular interest is identifying biomarkers in early life which can distinguish between early-onset wheezers who persist and those with transient symptoms. Early studies investigated eosinophilia as a potential biomarker[68-72]. One study showed variations in immune response within seemingly homogeneous IgE-mediated asthma phenotypes[73]. Differences in T-cell immunity response pattern may be markers of different airway disease endotypes. Non-invasive biomarkers, which are more suitable for infants, may be more appropriate for distinguishing between different asthma endotypes. Recently, a study has been set-up specifically aimed at making reliable asthma diagnosis as early as age two to three years using non-invasive biomarkers such as gases and non-volatile inflammatory markers such as cytokines, chemokines and epithelial lining in exhaled breath[74]. In future, non-invasive biomarkers are likely to be important in early diagnosis and possibly, prevention of asthma[75].

Fraction of Exhaled Nitric Oxide (FeNO), indicative of Th2-type airway inflammation, has been proposed as a potential biomarker for distinguishing endotypes [76-79] in early life due to its noninvasive nature[76,80-84]. However, FeNO is strongly correlated with atopy[85-88]. Studies have found that this biomarker can be used as a biomarker for transient wheeze but not for persistent wheeze phenotypes. As early as age one month, an elevated exhaled FeNO was seen in children who had recurrent wheeze in the first year of life but not thereafter[77]. FeNO is therefore likely a marker of airway inflammation and contemporaneous recurrent wheeze rather than a marker of distinct asthma endotypes.

With the advent of systems biology which combines mathematical computation with proteomics, genomics and other biological markers, research in identifying endotypes of asthma[89] has moved towards a more integrated approach to understanding interactions of these mechanisms in an attempt to identify more definitive asthma endotypes[90]. This has been described in the recent literature as the "-omics" approach which uses systems biology by combining potential biomarkers 
in high dimensional datasets using mathematical modelling techniques to reduce high throughput data into a lower dimensional space. This is a current area of research in computer and mathematical science. Such research may provide a more holistic approach for identifying more precise diagnostic strategies for understanding different endotypes[91,92]. However, these approaches present many challenges. Firstly, such studies need large sample sizes in order to have sufficient power in order to identify biological networks associated with different endotypes. Although the cost associated with eliciting such high throughput data is currently decreasing, funding can still be an issue for such studies. However, the trade-off of that cost would be greater efficiency in identifying more targeted treatment strategies.

\section{Expert Commentary: Clinical Implications of the Asthma Endotype}

One may ask whether wheeze phenotypes identified are clinically relevant and whether these have implications for clinical practice. To try to assign children who attend clinic to the wheeze phenotypes described in the birth cohorts is neither possible nor desirable. The assumption underlying the work reviewed is that asthma is not a single disease, but a group of heterogeneous diseases, with different profiles of symptom manifestations in childhood. The rationale of the computer-based approaches of pattern recognition reviewed is to better classify symptomatic children into groups with common features and ultimately to identify endotypes of asthma. It is anticipated that different endotypes will have unique underlying pathophysiological mechanisms (and underlying genetic and environmental predictors). The clinical relevance of this is that different "asthmas" may respond differently to different treatments, which will eventually allow us to move away from a "one size fits all" approach towards more targeted personalised or stratified therapies [46]. This is clinically relevant as one of the main challenges for asthma treatment in children remains the lack of understanding as to who will benefit in the long term from currently available treatments.[93,94]. However, we are still merely scratching the surface of this problem, and much remains to be done in making the leap from defining phenotypes to identifying distinct biological 
characteristics and mechanisms of endotypes. Therefore it would be premature to try to apply any of this work to current clinical practice.

\section{Five-year View: The Future of A More Unified Definition of the Asthma}

\section{Endotype}

Since the seminal paper in 1995 from Martinez et al, in which wheeze phenotypes in early life were first described, we have made considerable progress in developing computational techniques to describe patterns of wheeze and other asthma associated symptoms in early life. Although the literature reviewed shows some disparity in defining phenotypes of early life wheeze, there are many common features. However, much work needs to be done to progress to endotypes of asthma, with implications for personalized treatments and also preventative strategies. As seen from the small number of studies reviewed, few studies have addressed the question of establishing asthma phenotypes in a consistent manner. This points towards a need for more longitudinal birth cohorts in order to replicate possible genetic and environmental factors associated with different asthma phenotypes.

Computer-assisted reasoning can facilitate research in identifying asthma endotypes and their underlying biological mechanisms, capitalising on the wealth of data available from longitudinal birth cohorts. Statistical machine learning approaches can take examples of many profiles of the natural history of wheeze in order to apply generalisations of the profiles of wheeze on a wider population. By applying these techniques, the 'best' endotypes across multiple birth cohorts with similar characteristics can be identified. Similar to the consortia developed to power genome wide association studies, taking account of between cohort heterogeneity, integrating data on the natural history of wheeze from different settings would mean obtaining a more complete picture of the heterogeneity of asthma on a world-wide population, and thus will help us to make better inference. The asthma endotype is a hypothetical construct which has a tangible value in helping us to better 
understand the natural history of asthma disease-related diseases and therefore identify more effective personalised treatment strategies.

\section{Key Issues}

- Asthma is not a single disease but rather represents an umbrella term used to describe a collection of heterogeneous diseases presenting with similar symptoms.

- Longitudinal birth cohorts have been crucial for conceptualising the natural history of wheeze, the most common manifestation of asthma.

- Both subjective clinician-imposed and objective data-driven approaches have been used to capture changing trajectories of wheeze profiles over time in order to identify different asthma phenotypes.

- Computer-based objective data-driven approaches can help achieve a more refined clinicianbased diagnosis by understanding the different types of asthma phenotypes and their associated genetic and environmental characteristics.

- Further work is required to refine the phenotypes currently suggested and identify their genetic and environmental associates.

- By understanding the different phenotypes present in this umbrella term "asthma", we will better understand biological causes, thus facilitating personalised medicine including management and prevention strategies. 


\section{References}

1. Bousquet J, Clark TJ, Hurd S et al. GINA guidelines on asthma and beyond. Allergy 62(2), 102112 (2007).

2. Van Wonderen KE, Van Der Mark LB, Mohrs J, Bindels PJ, Van Aalderen WM, Ter Riet G. Different definitions in childhood asthma: how dependable is the dependent variable? Eur Respir J 36(1), 48-56 (2010).

3. Murray CS, Simpson A, Custovic A. Allergens, viruses, and asthma exacerbations. Proc Am Thorac Soc 1(2), 99-104 (2004).

4. Papadopoulos NG, Arakawa H, Carlsen KH et al. International consensus on (ICON) pediatric asthma. Allergy 67(8), 976-997 (2012).

** Gives an important evaluation of the current status of childhood asthma phenotypes and highlights the practical impliactions of understanding the asthma phneoytpe better.

5. Bosse Y, Hudson TJ. Toward a comprehensive set of asthma susceptibility genes. Annu Rev Med 58, 171-184 (2007).

6. Murk W, Walsh K, Hsu LI, Zhao L, Bracken MB, Dewan AT. Attempted replication of 50 reported asthma risk genes identifies a SNP in RAD50 as associated with childhood atopic asthma. Hum Hered 71(2), 97-105 (2011).

7. Hagerhed-Engman L, Bornehag CG, Sundell J, Aberg N. Day-care attendance and increased risk for respiratory and allergic symptoms in preschool age. Allergy 61(4), 447-453 (2006).

8. Nicolaou NC, Simpson A, Lowe LA, Murray CS, Woodcock A, Custovic A. Day-care attendance, position in sibship, and early childhood wheezing: a population-based birth cohort study. $J$ Allergy Clin Immunol 122(3), 500-506 (2008).

9. Nafstad P, Brunekreef B, Skrondal A, Nystad W. Early respiratory infections, asthma, and allergy: 10-year follow-up of the Oslo Birth Cohort. Pediatrics 116(2), e255-262 (2005).

10. Ober C, Vercelli D. Gene-environment interactions in human disease: nuisance or opportunity? Trends Genet 27(3), 107-115 (2011).

11. Simpson A, John SL, Jury F et al. Endotoxin exposure, CD14, and allergic disease: an interaction between genes and the environment. Am J Respir Crit Care Med 174(4), 386-392 (2006).

12. Martinez FD. Gene-environment interactions in asthma: with apologies to William of Ockham. Proc Am Thorac Soc 4(1), 26-31 (2007).

** Part of the complexity in defining and understanding risk factors associated with different asthma endotypes is the complexity of gene-environmental interactions which interplay during development stages in life

13. Lotvall J, Akdis CA, Bacharier LB et al. Asthma endotypes: a new approach to classification of disease entities within the asthma syndrome. J Allergy Clin Immunol 127(2), 355-360 (2011).

14. Wilson NM. Wheezy bronchitis revisited. Arch Dis Child 64(8), 1194-1199 (1989).

15. Wright AL, Taussig LM. Lessons from long-term cohort studies. Childhood asthma. Eur Respir J Supp/ 27, 17s-22s (1998).

16. Martinez FD. Development of wheezing disorders and asthma in preschool children. Pediatrics 109(2 Suppl), 362-367 (2002).

17. Hancox RJ, Subbarao P, Sears MR. Relevance of birth cohorts to assessment of asthma persistence. Curr Allergy Asthma Rep 12(3), 175-184 (2012).

18. Silverman M, Wilson N. Wheezing phenotypes in childhood. Thorax. 1997 Nov;52(11):936-7., 
19. Martinez FD. Genes, environments, development and asthma: a reappraisal. Eur Respir J 29(1), 179-184 (2007).

20. Patino $\mathrm{CM}$, Martinez FD. Interactions between genes and environment in the development of asthma. Allergy 56(4), 279-286 (2001).

21. Herzog R, Cunningham-Rundles S. Pediatric asthma: natural history, assessment, and treatment. Mt Sinai J Med 78(5), 645-660 (2011).

22. Stochl J, Croudace T, Perez J et al. Usefulness of EQ-5D for evaluation of health-related quality of life in young adults with first-episode psychosis. Qual Life Res 17, 17 (2012).

23. Colman I, Ploubidis GB, Wadsworth ME, Jones PB, Croudace TJ. A longitudinal typology of symptoms of depression and anxiety over the life course. Biol Psychiatry 62(11), 1265-1271 (2007).

24. Abbott RA, Ploubidis GB, Huppert FA, Kuh D, Wadsworth ME, Croudace TJ. Psychometric evaluation and predictive validity of Ryff's psychological well-being items in a UK birth cohort sample of women. Health Qual Life Outcomes 4, 76 (2006).

25. Barnett JH, Croudace TJ, Jaycock S et al. Improvement and decline of cognitive function in schizophrenia over one year: a longitudinal investigation using latent growth modelling. BMC Psychiatry 7, 16 (2007).

26. Pickles A, Bolton $\mathrm{P}, \mathrm{Macdonald} \mathrm{H}$ et al. Latent-class analysis of recurrence risks for complex phenotypes with selection and measurement error: a twin and family history study of autism. Am J Hum Genet 57(3), 717-726 (1995).

27. Martinez FD, Wright AL, Taussig LM, Holberg CJ, Halonen M, Morgan WJ. Asthma and wheezing in the first six years of life. The Group Health Medical Associates. N Engl J Med 332(3), 133-138 (1995).

** Proposes the existence of distinct wheeze phenotypes with different clinical associations

28. Lowe LA, Simpson A, Woodcock A, Morris J, Murray CS, Custovic A. Wheeze phenotypes and lung function in preschool children. Am J Respir Crit Care Med 171(3), 231-237 (2005).

29. Kurukulaaratchy RJ, Fenn MH, Waterhouse LM, Matthews SM, Holgate ST, Arshad SH. Characterization of wheezing phenotypes in the first 10 years of life. Clin Exp Allergy 33(5), 573-578 (2003).

30. Lau S, Illi S, Sommerfeld C et al. Transient early wheeze is not associated with impaired lung function in 7-yr-old children. Eur Respir J 21(5), 834-841 (2003).

31. Midodzi WK, Rowe BH, Majaesic CM, Saunders LD, Senthilselvan A. Predictors for wheezing phenotypes in the first decade of life. Respirology 13(4), 537-545 (2008).

32. Sherriff A, Peters TJ, Henderson J, Strachan D. Risk factor associations with wheezing patterns in children followed longitudinally from birth to 3(1/2) years. Int J Epidemiol 30(6), 1473-1484 (2001).

33. Turner SW, Palmer L, Rye PJ et al. The relationship between infant airway function, childhood airway responsiveness, and asthma. Am J Respir Crit Care Med 169(8), 921-927 (2004).

34. Henderson J, Granell R, Heron J et al. Associations of wheezing phenotypes in the first 6 years of life with atopy, lung function and airway responsiveness in mid-childhood. Thorax 63(11), 974-980 (2008). 
** First paper to use a data-driven approach to identify distinct wheeze phenotypes using a data-driven approach (latent class analysis) to understand the natural history of wheeze over time.

35. Savenije OE, Granell R, Caudri D et al. Comparison of childhood wheezing phenotypes in 2 birth cohorts: ALSPAC and PIAMA. J Allergy Clin Immunol 127(6), 1505-1512 (2011).

36. Granell R, Sterne JA, Henderson J. Associations of different phenotypes of wheezing illness in early childhood with environmental variables implicated in the aetiology of asthma. PLoS One 7(10), 31 (2012).

37. Chen $Q$, Just AC, Miller RL et al. Using latent class growth analysis to identify childhood wheeze phenotypes in an urban birth cohort. Ann Allergy Asthma Immunol 108(5), 311-315 (2012).

38. Lowe L, Murray CS, Martin L et al. Reported versus confirmed wheeze and lung function in early life. Arch Dis Child 89(6), 540-543 (2004).

39. Pickles A, Croudace T. Latent mixture models for multivariate and longitudinal outcomes. Stat Methods Med Res 19(3), 271-289 (2009).

40. Belgrave DCM, Simpson A, Semic-Jusufagic A et al. Joint modeling of parentally-reported and physician-confirmed wheeze identifies children with persistent troublesome wheezing. J Allergy Clin Immunol (2013).

** Incorporates physician-reported and parentally-reported wheeze to define a novel class of wheezers who have poor response to treatment and have distinct physiological characteristics

41. Collins SA, Pike KC, Inskip HM et al. Validation of novel wheeze phenotypes using longitudinal airway function and atopic sensitization data in the first 6 years of life: Evidence from the Southampton Women's survey. Pediatr Pulmonol 8(10), 22766 (2013).

**Comparison of data- and hypothesis- driven approaches to identifying wheeze phenotypes carried out within the same population

42. Von Mutius E. Trajectories of childhood wheeze. J Allergy Clin Immunol. 2011 Jun;127(6):1513-4. doi: 10.1016/j.jaci.2011.04.026. Epub 2011 May 4.,

43. Castro-Rodriguez JA, Garcia-Marcos L. Wheezing and Asthma in childhood: an epidemiology approach. Allergol Immunopathol 36(5), 280-290 (2008).

44. Bosco A, Holt PG. Genome-wide expression profiling of T-cells in childhood wheeze. Eur Respir J. 2008 Nov;32(5):1138-40. doi: 10.1183/09031936.00130108.,

45. Scott M, Kurukulaaratchy RJ, Raza A, Arshad SH. Understanding the nature and outcome of childhood wheezing. Eur Respir J. 2009 Mar;33(3):700-1. doi: 10.1183/09031936.00149408.,

46. Kurukulaaratchy RJ, Waterhouse L, Matthews SM, Arshad SH. Are influences during pregnancy associated with wheezing phenotypes during the first decade of life? Acta Paediatr 94(5), 553-558 (2005).

47. Stein RT, Martinez FD. Asthma phenotypes in childhood: lessons from an epidemiological approach. Paediatr Respir Rev 5(2), 155-161 (2004).

48. Henderson J, Hilliard TN, Sherriff A, Stalker D, Al Shammari N, Thomas HM. Hospitalization for RSV bronchiolitis before 12 months of age and subsequent asthma, atopy and wheeze: a longitudinal birth cohort study. Pediatr Allergy Immunol 16(5), 386-392 (2005).

49. Stein RT, Sherrill D, Morgan WJ et al. Respiratory syncytial virus in early life and risk of wheeze and allergy by age 13 years. Lancet 354(9178), 541-545 (1999). 
50. Jackson DJ, Gangnon RE, Evans $\mathrm{MD}$ et al. Wheezing rhinovirus illnesses in early life predict asthma development in high-risk children. Am J Respir Crit Care Med 178(7), 667-672 (2008).

51. Stein RT, Martinez FD. Respiratory syncytial virus and asthma: still no final answer. Thorax 65(12), 1033-1034

52. Murray CS, Woodcock A, Langley SJ, Morris J, Custovic A. Secondary prevention of asthma by the use of Inhaled Fluticasone propionate in Wheezy INfants (IFWIN): double-blind, randomised, controlled study. Lancet 368(9537), 754-762 (2006).

53. Bisgaard H, Hermansen MN, Loland L, Halkjaer LB, Buchvald F. Intermittent inhaled corticosteroids in infants with episodic wheezing. N Engl J Med 354(19), 1998-2005 (2006).

54. Ducharme FM, Lemire C, Noya FJ et al. Preemptive use of high-dose fluticasone for virusinduced wheezing in young children. N Engl J Med 360(4), 339-353 (2009).

55. Simpson A, Tan VY, Winn J et al. Beyond atopy: multiple patterns of sensitization in relation to asthma in a birth cohort study. Am J Respir Crit Care Med 181(11), 1200-1206 (2010).

56. Lazic N, Roberts $\mathrm{G}$, Custovic $\mathrm{A}$ et al. Multiple atopy phenotypes and their associations with asthma: similar findings from two birth cohorts. Allergy 68(6), 764-770 (2013).

57. Wright AL. Analysis of epidemiological studies: facts and artifacts. Paediatr Respir Rev 3(3), 198-204 (2002).

58. Smith JA, Drake R, Simpson A, Woodcock A, Pickles A, Custovic A. Dimensions of respiratory symptoms in preschool children: population-based birth cohort study. Am J Respir Crit Care Med 177(12), 1358-1363 (2008).

** Uses principal component analysis to identify different dimensions of respiratory disease. This study helps to answer the question: what are the important questions for determining different respiratory diseases?

59. Spycher BD, Silverman M, Brooke AM, Minder CE, Kuehni CE. Distinguishing phenotypes of childhood wheeze and cough using latent class analysis. Eur Respir J 31(5), 974-981 (2008).

60. Spycher BD, Silverman M, Barben J et al. A disease model for wheezing disorders in preschool children based on clinicians' perceptions. PLoS One 4(12), 0008533 (2009).

61. Brand PL, Baraldi E, Bisgaard $\mathrm{H}$ et al. Definition, assessment and treatment of wheezing disorders in preschool children: an evidence-based approach. Eur Respir J 32(4), 1096-1110 (2008).

62. Fitzpatrick AM, Teague WG, Meyers DA et al. Heterogeneity of severe asthma in childhood: confirmation by cluster analysis of children in the National Institutes of Health/National Heart, Lung, and Blood Institute Severe Asthma Research Program. J Allergy Clin Immunol 127(2), 382-389 (2011).

63. Just J, Gouvis-Echraghi R, Rouve S, Wanin S, Moreau D, Annesi-Maesano I. Two novel, severe asthma phenotypes identified during childhood using a clustering approach. Eur Respir J 40(1), 55-60 (2012).

64. Herr M, Just J, Nikasinovic L et al. Risk factors and characteristics of respiratory and allergic phenotypes in early childhood. J Allergy Clin Immunol 130(2), 389-396 (2012).

65. Castro-Rodriguez JA. The Asthma Predictive Index: a very useful tool for predicting asthma in young children. J Allergy Clin Immunol 126(2), 212-216 (2010).

66. Matricardi PM, Illi S, Keil T, Wagner P, Wahn U, Lau S. Predicting persistence of wheezing: one algorithm does not fit all. Eur Respir J. 2010 Mar;35(3):701-3. doi: 10.1183/09031936.00163709., 
67. Caudri D, Wijga A, Cm AS et al. Predicting the long-term prognosis of children with symptoms suggestive of asthma at preschool age. J Allergy Clin Immunol 124(5), 903-910 (2009).

68. Walker $\mathrm{C}$, Kagi MK, Ingold $\mathrm{P}$ et al. Atopic dermatitis: correlation of peripheral blood $\mathrm{T}$ cell activation, eosinophilia and serum factors with clinical severity. Clin Exp Allergy 23(2), 145153 (1993).

69. Hughes JM, Rimmer SJ, Salome CM et al. Eosinophilia, interleukin-5, and tumour necrosis factor-alpha in asthmatic children. Allergy 56(5), 412-418 (2001).

70. Klion AD, Law MA, Riemenschneider W et al. Familial eosinophilia: a benign disorder? Blood 103(11), 4050-4055 (2004).

71. Just J, Nicoloyanis N, Chauvin M, Pribil C, Grimfeld A, Duru G. Lack of eosinophilia can predict remission in wheezy infants? Clin Exp Allergy 38(5), 767-773 (2008).

72. Sacco O, Sale R, Silvestri $M$ et al. Total and allergen-specific IgE levels in serum reflect blood eosinophilia and fractional exhaled nitric oxide concentrations but not pulmonary functions in allergic asthmatic children sensitized to house dust mites. Pediatr Allergy Immunol 14(6), 475-481 (2003).

73. Heaton $\mathrm{T}$, Rowe J, Turner $\mathrm{S}$ et al. An immunoepidemiological approach to asthma: identification of in-vitro T-cell response patterns associated with different wheezing phenotypes in children. Lancet 365(9454), 142-149 (2005).

74. Van De Kant KD, Klaassen EM, Jobsis Q, Nijhuis AJ, Van Schayck OC, Dompeling E. Early diagnosis of asthma in young children by using non-invasive biomarkers of airway inflammation and early lung function measurements: study protocol of a case-control study. BMC Public Health 9(210), 1471-2458 (2009).

75. Hauk PJ, Krawiec M, Murphy J et al. Neutrophilic airway inflammation and association with bacterial lipopolysaccharide in children with asthma and wheezing. Pediatr Pulmonol 43(9), 916-923 (2008).

76. Van Der Valk RJ, Caudri D, Savenije $\mathrm{O}$ et al. Childhood wheezing phenotypes and FeNO in atopic children at age 8. Clin Exp Allergy 42(9), 1329-1336 (2012).

77. Chawes BL, Buchvald F, Bischoff AL et al. Elevated exhaled nitric oxide in high-risk neonates precedes transient early but not persistent wheeze. Am J Respir Crit Care Med 182(2), 138142 (2010).

78. Olin AC, Rosengren A, Thelle DS, Lissner L, Toren K. Increased fraction of exhaled nitric oxide predicts new-onset wheeze in a general population. Am J Respir Crit Care Med 181(4), 324327 (2010).

79. Bastain TM, Islam T, Berhane KT et al. Exhaled nitric oxide, susceptibility and new-onset asthma in the Children's Health Study. Eur Respir J 37(3), 523-531 (2011).

80. Bates CA, Silkoff PE. Exhaled nitric oxide in asthma: from bench to bedside. J Allergy Clin Immunol 111(2), 256-262 (2003).

81. Paredi P, Kharitonov SA, Barnes PJ. Analysis of expired air for oxidation products. Am J Respir Crit Care Med 166(12 Pt 2), S31-37 (2002).

82. Von Mutius E, Illi S, Hirsch T, Leupold W, Keil U, Weiland SK. Frequency of infections and risk of asthma, atopy and airway hyperresponsiveness in children. Eur Respir J 14(1), 4-11 (1999).

83. Baraldi E, De Jongste JC. Measurement of exhaled nitric oxide in children, 2001. Eur Respir J 20(1), 223-237 (2002). 
84. Kharitonov SA, Barnes PJ. Exhaled markers of pulmonary disease. Am J Respir Crit Care Med 163(7), 1693-1722 (2001).

85. Linn WS, Rappaport EB, Berhane KT, Bastain TM, Avol EL, Gilliland FD. Exhaled nitric oxide in a population-based study of southern California schoolchildren. Respir Res 10(28), 14659921 (2009).

86. Gratziou C, Lignos M, Dassiou M, Roussos C. Influence of atopy on exhaled nitric oxide in patients with stable asthma and rhinitis. Eur Respir J 14(4), 897-901 (1999).

87. Franklin PJ, Turner SW, Le Souef PN, Stick SM. Exhaled nitric oxide and asthma: complex interactions between atopy, airway responsiveness, and symptoms in a community population of children. Thorax 58(12), 1048-1052 (2003).

88. Prasad A, Langford B, Stradling JR, Ho LP. Exhaled nitric oxide as a screening tool for asthma in school children. Respir Med 100(1), 167-173 (2006).

89. Hopkin JM. The diagnosis of asthma, a clinical syndrome. Thorax 67(7), 660-662 (2012).

90. Sittka A, Vera J, Lai X, Schmeck BT. Asthma phenotyping, therapy, and prevention: what can we learn from systems biology? Pediatr Res 73(4 Pt 2), 543-552 (2013).

91. Calvo FQ, Fillet $\mathrm{M}$, De Seny $\mathrm{D}$ et al. Biomarker discovery in asthma-related inflammation and remodeling. Proteomics 9(8), 2163-2170 (2009).

92. O'neil SE, Sitkauskiene B, Babusyte A et al. Network analysis of quantitative proteomics on asthmatic bronchi: effects of inhaled glucocorticoid treatment. Respir Res 12(124), 14659921 (2011).

93. Schultz A, Brand PL. Phenotype-directed treatment of pre-school-aged children with recurrent wheeze. J Paediatr Child Health 48(2), 1440-1754 (2012).

94. Van Aalderen WM, Sprikkelman AB. Inhaled corticosteroids in childhood asthma: the story continues. Eur J Pediatr 170(6), 709-718 (2011). 
Association With Early Impairment Lung Function (Infancy)

Cohorts using a Data-driven approach to define Wheeze Phenotypes

ALSPAC

Transient-Early Wheeze Persistent Wheeze Late-Onset Wheeze

PIAMA

CCCEH

Not Done

Not Done

MAAS

Not Done

Cohorts using an Investigator-Imposed approach to define Wheeze Phenotypes

Transient-Early Wheeze Persistent Wheeze Late-Onset Wheeze

TCRS $\quad \uparrow \quad$ No Association No Association

ALSPAC Not Done

Prolonged Early Wheeze Intermediate-Onset Wheeze Persistent Troublesome Wheeze Ref

SWS (ALSPAC)

No Association

No Association

个

No Association

Association With Lung Function Impairment by Age 6

Cohorts using a Data-driven approach to define Wheeze Phenotype

ALSPAC

PIAMA

Transient-Early Wheeze Persistent Wheeze Late-Onset Wheeze

No Association

Not Done

$\uparrow$

$\uparrow$
Prolonged Early Wheeze Intermediate-Onset Wheeze Persistent Troublesome Wheeze Ref $\uparrow$

No Association

[34]

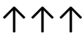
$\frac{\text { Cohorts using an Investigator-Imposed approach to define Wheeze Phenotypes }}{\text { Transient-Early Wheeze Persistent Wheeze Late-Onset Wheeze }}$

TCRS
No Association
No Association Not Done

Prolonged Early Wheeze Intermediate-Onset Wheeze

MAAS $\uparrow$

$\uparrow \uparrow$

SWS (ALSPAC) No Association $\quad \uparrow \quad$ No Association

SWS (TCRS) No Association $\quad \uparrow \quad$ No Association

Association With Early Impairment Broncho-hyperresponsiveness

Cohorts using a Data-driven approach to define Wheeze Phenotypes

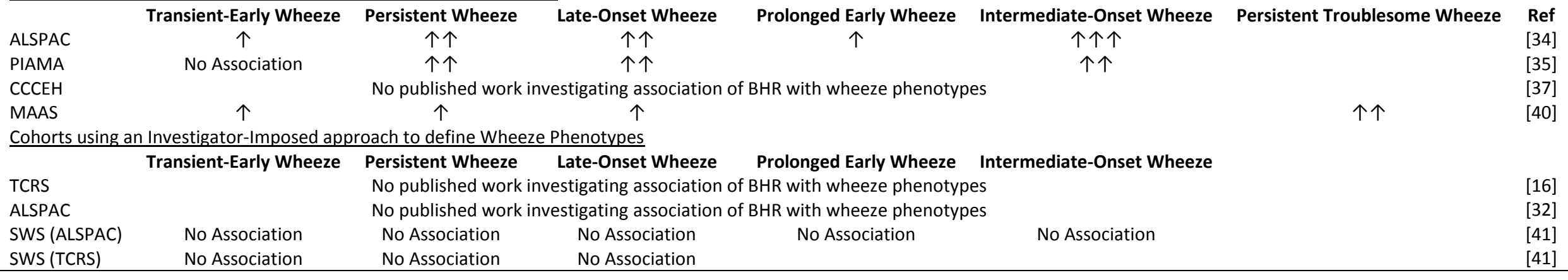




\begin{tabular}{|c|c|c|c|c|c|c|c|}
\hline \multicolumn{8}{|c|}{ Association With IgE } \\
\hline \multicolumn{8}{|c|}{ Cohorts using a Data-driven approach to define Wheeze Phenotypes } \\
\hline & Transient-Early Wheeze & Persistent Wheeze & Late-Onset Wheeze & Prolonged Early Wheeze & Intermediate-Onset Wheeze & Persistent Troublesome Wheeze & Ref \\
\hline ALSPAC & \multicolumn{6}{|c|}{ No published work investigating association of IgE with wheeze phenotypes } & [34] \\
\hline PIAMA & No Association & 个 (only at age 8) & 个个 (age 4 and 8 years) & & 个个 (age 4 and 8 years) & & [35] \\
\hline CCCEH & \multicolumn{6}{|c|}{ Not Done } & [37] \\
\hline MAAS & No Association & $\uparrow \uparrow$ & $\uparrow \uparrow$ & & & 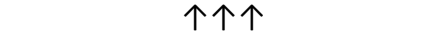 & [40] \\
\hline \multicolumn{8}{|c|}{ Investigator-Imposed approach to define Wheeze Phenotypes } \\
\hline & Transient-Early Wheeze & Persistent Wheeze & \multicolumn{5}{|l|}{ Late-Onset Wheeze } \\
\hline TCRS & No Association & 个个 & & & & & [16] \\
\hline ALSPAC & \multicolumn{6}{|c|}{ No published work investigating association of IgE with wheeze phenotypes } & [32] \\
\hline SWS & \multicolumn{6}{|c|}{ No published work investigating association of IgE with wheeze phenotypes } & [41] \\
\hline \multicolumn{8}{|c|}{ Association With Sensitisation (Skin Prick Test) } \\
\hline \multicolumn{8}{|c|}{ Cohorts using a Data-driven approach to define Wheeze Phenotypes } \\
\hline & Transient-Early Wheeze & Persistent Wheeze & Late-Onset Wheeze & Prolonged Early Wheeze & Intermediate-Onset Wheeze & Persistent Troublesome Wheeze & Ref \\
\hline ALSPAC & No Association & 个个 & 个个 & No Association & 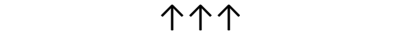 & & [34] \\
\hline PIAMA & \multicolumn{6}{|c|}{ No published work investigating association of SPT with wheeze phenotypes } & [35] \\
\hline CCCEH & \multicolumn{6}{|c|}{ No published work investigating association of SPT with wheeze phenotypes } & [37] \\
\hline MAAS & No Association & $\uparrow$ & $\uparrow$ & & & $\uparrow \uparrow$ & [40] \\
\hline \multicolumn{8}{|c|}{ Cohorts using an Investigator-Imposed approach to define Wheeze Phenotypes } \\
\hline & Transient-Early Wheeze & Persistent Wheeze & Late-Onset Wheeze & Prolonged Early Wheeze & Intermediate-Onset Wheeze & & \\
\hline TCRS & No Association & $\uparrow \uparrow$ & $\uparrow \uparrow$ & & & & [16] \\
\hline ALSPAC & \multicolumn{6}{|c|}{ No published work investigating association of SPT with wheeze phenotypes } & [32] \\
\hline SWS(ALSPAC) & No Association & 个个 (age 3, 6) & 个个 (age 3，6) & No Association & 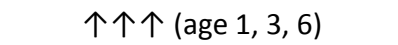 & & [41] \\
\hline SWS (TCRS) & $\downarrow$ & 个个 (age 1, 3, 6) & 个个 (age 3,6) & & & & [41] \\
\hline
\end{tabular}

Table 3a: Characteristics of different wheeze phenotypes identified by different longitudinal birth cohorts 


\section{Association With Smoking}

Cohorts using a Data-driven approach to define Wheeze Phenotypes

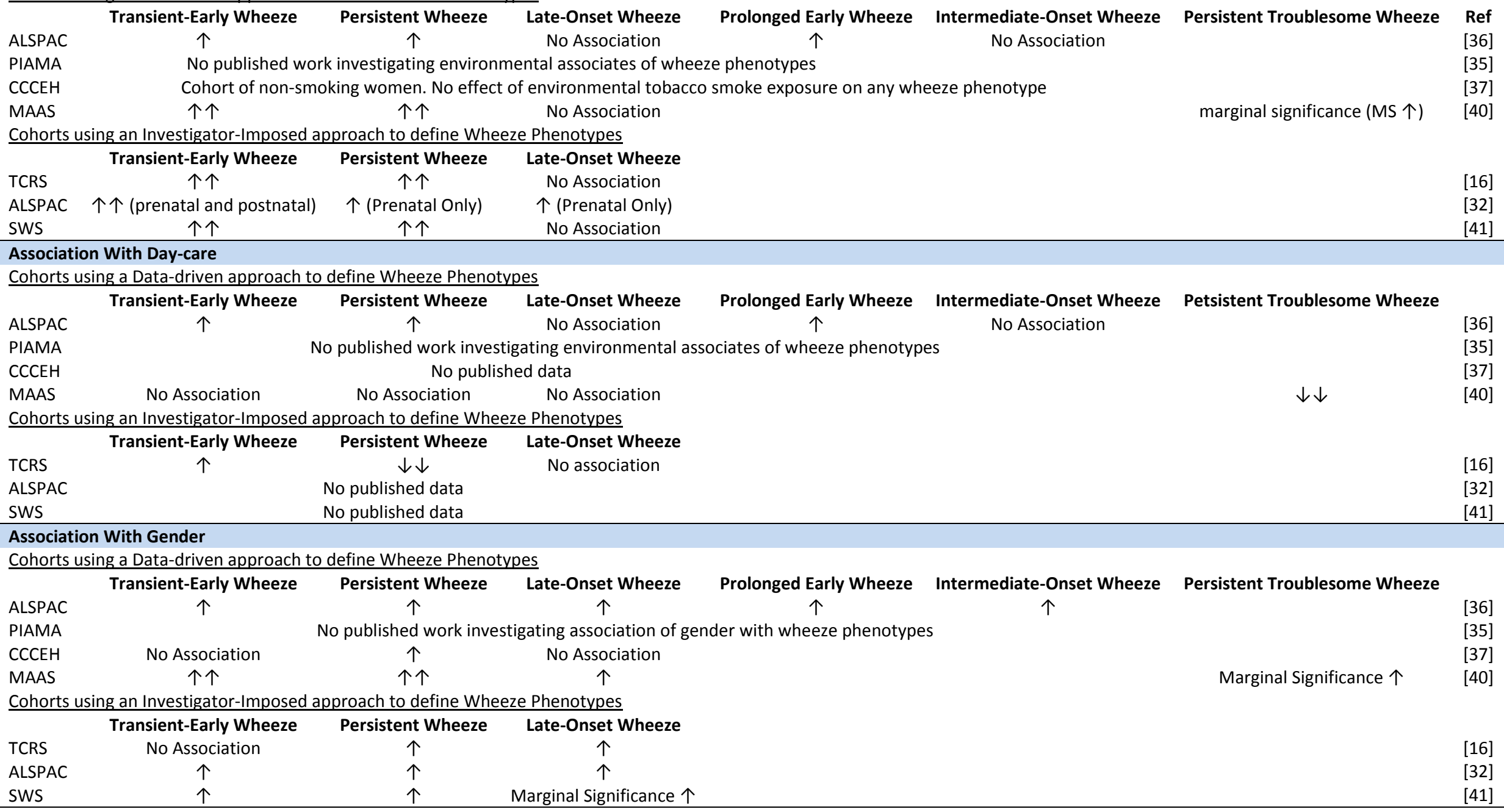




\section{Association With Older Siblings}

Cohorts using a Data-driven approach to define Wheeze Phenotypes

\begin{tabular}{|c|c|c|c|c|c|c|c|}
\hline ALSPAC & $\begin{array}{c}\text { Transient-Early Wheeze } \\
\uparrow\end{array}$ & $\begin{array}{c}\text { Persistent Wheeze } \\
\uparrow\end{array}$ & $\begin{array}{l}\text { Late-Onset Wheeze } \\
\text { No Association }\end{array}$ & $\begin{array}{l}\text { Prolonged Early Wheeze } \\
\text { No Association }\end{array}$ & $\begin{array}{c}\text { Intermediate-Onset Wheeze } \\
\text { No Association }\end{array}$ & Persistent Troublesome Wheeze & $\begin{array}{l}\text { Ref } \\
{[36]}\end{array}$ \\
\hline PIAMA & & o published work inv & stigating association o & sibship with wheeze pheno & types & & [35] \\
\hline CCCEH & & o published work inv & stigating association o & sibship with wheeze pheno & types & & [37] \\
\hline MAAS & No Association & $\downarrow \downarrow$ & No Association & & & $\downarrow$ Marginally Significant & [40] \\
\hline \multicolumn{8}{|c|}{ Cohorts using an Investigator-Imposed approach to define Wheeze Phenotypes } \\
\hline & Transient-Early Wheeze & Persistent Wheeze & Late-Onset Wheeze & & & & \\
\hline TCRS & $\uparrow$ & $\downarrow \downarrow$ & No Association & & & & [16] \\
\hline ALSPAC & 个个 & $\uparrow$ & $\uparrow$ & & & & [32] \\
\hline SWS & & & No published d & & & & [41] \\
\hline \multicolumn{8}{|c|}{ Association With BMI/ Weight } \\
\hline \multicolumn{8}{|c|}{ Cohorts using a Data-driven approach to define Wheeze Phenotypes } \\
\hline & Transient-Early Wheeze & Persistent Wheeze & Late-Onset Wheeze & Prolonged Early Wheeze & Intermediate-Onset Wheeze & & \\
\hline ALSPAC: Low birth weight & & & & & $\uparrow$ & & [36] \\
\hline PIAMA & No published wo & $\mathrm{k}$ investigating associ & ation of Weight with wl & eeze phenotypes & & & [35] \\
\hline CCCEH & No published wo & $\mathrm{k}$ investigating associ & tion of Weight with wl & leeze phenotypes & & & [37] \\
\hline MAAS & No published wo & $\mathrm{k}$ investigating associ & tion of Weight with wl & eeze phenotypes & & & [40] \\
\hline \multicolumn{8}{|c|}{ Cohorts using an Investigator-Imposed approach to define Wheeze Phenotypes } \\
\hline (2) & Transient-Early Wheeze & Persistent Wheeze & Late-Onset Wheeze & & & & \\
\hline TCRS & \multirow{3}{*}{\multicolumn{6}{|c|}{$\begin{array}{l}\text { No published work investigating association of Weight with wheeze phenotypes } \\
\text { No Association No Association } \quad \uparrow \\
\text { No published work investigating association of Weight with wheeze phenotypes }\end{array}$}} & [16] \\
\hline ALSPAC: Low birth weight & & & & & & & [32] \\
\hline SWS & & & & & & & [41] \\
\hline \multicolumn{8}{|c|}{ Association With Maternal Asthma } \\
\hline \multicolumn{8}{|c|}{ Cohorts using a Data-driven approach to define Wheeze Phenotypes } \\
\hline & Transient-Early Wheeze & Persistent Wheeze & Late-Onset Wheeze & Prolonged Early Wheeze & Intermediate-Onset Wheeze & Persistent Controlled Wheeze & \\
\hline ALSPAC & $\uparrow$ & $\uparrow \uparrow$ & $\uparrow$ & $\uparrow$ & $\uparrow$ & & [34] \\
\hline PIAMA & \multicolumn{6}{|c|}{ No published work investigating association of Maternal Asthma with wheeze phenotypes } & [35] \\
\hline CCCEH & $\uparrow$ & 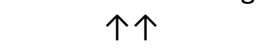 & $\uparrow$ & & & & [37] \\
\hline MAAS & $\uparrow$ & 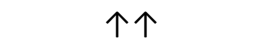 & 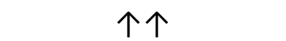 & & & Marginally Significant $\uparrow$ & [40] \\
\hline \multicolumn{8}{|c|}{ Cohorts using an Investigator-Imposed approach to define Wheeze Phenotypes } \\
\hline & Transient-Early Wheeze & Persistent Wheeze & Late-Onset Wheeze & & & & \\
\hline TCRS & No Association & $\uparrow$ & $\uparrow$ & & & & [16] \\
\hline ALSPAC & $\uparrow$ & 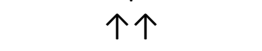 & $\uparrow$ & & & & [32] \\
\hline SWS & 个个 & 个个 & 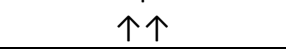 & & & & [41] \\
\hline
\end{tabular}

Table 3b: Risk factors associated with different wheeze phenotypes identified by different longitudinal birth cohorts 
